\title{
Channel-noise-induced critical slowing in the subthreshold Hodgkin-Huxley neuron
}

\author{
Alex Bukoski* \\ College of Veterinary Medicine, University of Missouri, Columbia, Missouri 65211, USA \\ D. A. Steyn-Ross ${ }^{\dagger}$ and Moira L. Steyn-Ross $\ddagger$ \\ School of Engineering, University of Waikato, Hamilton 3240, New Zealand \\ (Received 3 June 2013; revised manuscript received 2 December 2014; published 23 March 2015)
}

\begin{abstract}
The dynamics of a spiking neuron approaching threshold is investigated in the framework of Markov-chain models describing the random state-transitions of the underlying ion-channel proteins. We characterize subthreshold channel-noise-induced transmembrane potential fluctuations in both type-I (integrator) and type-II (resonator) parametrizations of the classic conductance-based Hodgkin-Huxley equations. As each neuron approaches spiking threshold from below, numerical simulations of stochastic trajectories demonstrate pronounced growth in amplitude simultaneous with decay in frequency of membrane voltage fluctuations induced by ion-channel state transitions. To explore this progression of fluctuation statistics, we approximate the exact Markov treatment with a 12-variable channel-based stochastic differential equation (SDE) and its Ornstein-Uhlenbeck (OU) linearization and show excellent agreement between Markov and SDE numerical simulations. Predictions of the OU theory with respect to membrane potential fluctuation variance, autocorrelation, correlation time, and spectral density are also in agreement and illustrate the close connection between the eigenvalue structure of the associated deterministic bifurcations and the observed behavior of the noisy Markov traces on close approach to threshold for both integrator and resonator point-neuron varieties.
\end{abstract}

DOI: 10.1103/PhysRevE.91.032708

PACS number(s): 87.19.L-, 05.10.Gg, 05.70.Fh, 87.17.Aa

\section{INTRODUCTION}

As described by Hodgkin-Huxley type models [1], the nonlinear dependence of ion-channel conductance on transmembrane voltage is the basis of action potential generation and provides a link between a neuron's subthreshold response and suprathreshold dynamics. Because membrane voltage perturbations from both deterministic and stochastic sources are known to significantly influence the computational properties of neurons, there is active interest in modeling these effects to better understand their consequences [2]. In the absence of noisy synaptic input, the most dominant source of intrinsic neuronal noise is due to thermally induced random transitions of ion channels between open and closed states [3]. Although seemingly less significant than extrinsic synaptic noise based on magnitude alone, ion-channel noise has been shown to influence the reliability and precision of action potential initiation [4] as well as the generation of spontaneous action potentials [5].

Although many models of neuronal excitability have been published, the classic Hodgkin-Huxley equations [1] retain a close connection to biological reality, are extremely robust, and - with appropriate parametrization — can reproduce nearly all observed single-neuron neurocomputational properties [6]. While the majority of these properties, defined by various spiking or bursting patterns, exist in the suprathreshold regime, there exist only two fundamental deterministic subthreshold behaviors as injected stimulus current crosses threshold: type-I excitability characterized by emergence of spiking oscillations at arbitrarily low frequency and type-II excitability wherein

\footnotetext{
*bukoskia@missouri.edu

†asr@waikato.ac.nz

${ }^{\ddagger}$ msr@waikato.ac.nz
}

oscillations emerge at finite frequency. This categorization of firing rate behavior at action potential onset is originally due to Hodgkin [7], though more recently Izhikevich [8] has introduced the terms integrator for type-I behavior and resonator for type-II behavior. Although action potentials emerge at threshold, these different firing rate behaviors arise from subthreshold properties dictated by the bifurcation structure of the deterministic system at spiking onset: In the case of type-I behavior the bifurcation is of saddle-node type, and in the case of type-II behavior the bifurcation is of subcritical Andronov-Hopf type.

Given the deterministic bifurcation structure of type-I and type-II neurons as they pass from the quiescent to spiking regimes, the theory of nonlinear dynamical systems predicts increasingly slow dynamics on close approach to threshold $[9,10]$. Within this framework, excursions from equilibrium and exploration of local phase space can be provoked via the addition of low-intensity driving noise using a stochastic differential equation (SDE) description [11]. Steyn-Ross et al. [12] have described growth of fluctuations and time scale prolongation in response to stochastic input in a reduced dimensionality point-neuron model on close approach to spiking threshold and identified this as critical slowing-down. For historical perspective, dynamical slowing was demonstrated in the squid giant axon preparation in 1978 by Matsumoto and Kunisawa [13] using changes in the extracellular bathing medium as the experimentally controlled bifurcation parameter. The authors demonstrate oscillatory exponential return to resting transmembrane potential following application of weak current pulses far from the transition to spiking and nonexponential dynamically slowed relaxation upon close approach to threshold. Although growth in amplitude of transmembrane potential oscillations in response to fixed input current magnitude as threshold is approached from below was not directly addressed in 
that paper, such growth appears to have been qualitatively observed.

In this paper we investigate the influence of ion-channel noise on the subthreshold dynamical behavior of two parametrizations of the Hodgkin-Huxley point-neuron model via numerical simulation of the underlying Markov-chain ion-channel models as the input current threshold for action potential generation is approached from below. We demonstrate critical slowing-down of the transmembrane potential fluctuation dynamics and compare these results to those of a 12-variable channel-based SDE model due to Fox and Lu [14]. We find that numerical simulations of the full nonlinear SDE, as well as predictions derived from its Ornstein-Uhlenbeck linearization, accurately capture the subthreshold fluctuation statistics of the detailed Markov-chain model. This progression of models-from Markov, to full SDE, and, finally, to linear SDE-demonstrates the close connection between the observed behavior of the Markov stochastic trajectories and the eigenvalue structure of the associated deterministic bifurcations on approach to threshold.

\section{MODELS}

The deterministic Hodgkin-Huxley point-neuron model is given by the following set of four nonlinear ordinary differential equations,

$$
\begin{aligned}
C \frac{d V}{d t}= & I_{\mathrm{DC}}-\bar{g}_{\mathrm{Na}} m^{3} h\left(V-E_{\mathrm{Na}}\right)-\bar{g}_{K} n^{4}\left(V-E_{\mathrm{K}}\right) \\
& -g_{L}\left(V-E_{L}\right) \\
\frac{d x}{d t}= & \alpha_{x}(V)(1-x)-\beta_{x}(V) x
\end{aligned}
$$

where $V$ is transmembrane potential measured with respect to the extracellular environment, $x \in\{n, m, h\}$, and $n, m$, and $h$ are gating variables that describe the kinetics of potassium channel activation, sodium channel activation, and sodium channel inactivation, respectively. The $\alpha_{x}(V)$ and $\beta_{x}(V)$ functions are transmembrane potential-dependent first-order rate constants for gate opening and closing; $C$ is membrane capacitance; $E_{\mathrm{Na}}, E_{\mathrm{K}}$, and $E_{L}$ are, respectively, the sodium, potassium, and leakage ion-channel reversal potentials; $I_{\mathrm{DC}}$ is an injected stimulus current which we treat as the bifurcation parameter; and $g_{L}$ is the leakage ion-channel conductance. The sodium and potassium channel conductances are given, respectively, by the $\bar{g}_{\mathrm{Na}} m^{3} h$ and $\bar{g}_{\mathrm{K}} n^{4}$ terms in Eq. (1a) where $\bar{g}_{\mathrm{Na}}$ and $\bar{g}_{\mathrm{K}}$ are the maximum conductances.

Two parametrizations of the Hodgkin-Huxley model characteristic of type-I and type-II excitabilities are explored in this paper. The first, taken from Nowotny and Rabinovich $[15,16]$, describes type-I integrator behavior and is based on a mammalian hippocampal pyramidal cell model of Traub and Miles [17]. Note that the parameters given in Refs. [15] and [16] have been scaled here to a standard specific capacitance value of $1 \mu \mathrm{F} \mathrm{cm}^{-2}$. The second describes type-II resonator behavior and is the original parameter set of Hodgkin and Huxley, appropriate to the squid giant axon at $6.3^{\circ} \mathrm{C}$ but modified such that $V$ represents the actual transmembrane potential measured with respect to the extracellular environment [18].
TABLE I. Hodgkin-Huxley model constants for the mammalian neuron model due to Nowotny [15,16] (column 2), which demonstrates type-I integrator behavior, and for the squid giant axon model due to Guevara [18] (column 3), which demonstrates type-II resonator behavior.

\begin{tabular}{lccc}
\hline \hline Symbol & Integrator & Resonator & Unit \\
\hline$C$ & 1 & 1 & $\mu \mathrm{F} \mathrm{cm}^{-2}$ \\
$E_{\mathrm{Na}}$ & 50 & 55 & $\mathrm{mV}$ \\
$E_{\mathrm{K}}$ & -95 & -72 & $\mathrm{mV}$ \\
$E_{L}$ & -63.563 & -49.387 & $\mathrm{mV}$ \\
$\bar{g}_{\mathrm{Na}}$ & 50 & 120 & $\mathrm{mS} \mathrm{cm}^{-2}$ \\
$\bar{g}_{\mathrm{K}}$ & 10 & 36 & $\mathrm{mS} \mathrm{cm}^{-2}$ \\
$g_{L}$ & 0.187 & 0.3 & $\mathrm{mS} \mathrm{cm}^{-2}$ \\
$I_{\mathrm{DC}}^{\text {crit }}$ & $\approx 0.35577$ & $\approx 9.7754$ & $\mu \mathrm{A} \mathrm{cm}^{-2}$ \\
$\rho_{\mathrm{Na}}$ & 60 & 60 & ${\mathrm{Channels} \mu \mathrm{m}^{-2}}$ \\
$\rho_{\mathrm{K}}$ & 18 & 18 & ${\text { Channels } \mu \mathrm{m}^{-2}}$ \\
$A$ & 3200 & 4000 & $\mu \mathrm{m}^{2}$ \\
\hline \hline
\end{tabular}

Parameter values for these two models are given in Table I and gating functions are given in Table II.

\section{A. Markov-chain ion-channel model}

Rather than treat the Hodgkin-Huxley potassium and sodium channel conductances of Eq. (1a) as deterministic, Markov-chain ion-channel models explicitly incorporate the underlying discrete state transitions, presumed to be the origin of channel noise, under the Markov assumption that state transitions are memoryless, identical, and independent. In this approach, Eq. (1b) are replaced by the potassium and sodium channel Markov chains (kinetic schemes) given in Figs. 1 and 2, respectively, with an algorithm for simulating the time between successive state transitions and for identifying the specific transition which occurs at that time. The deterministic channel conductances of Eq. (1a) are now time-dependent stochastic variables defined by

$$
\bar{g}_{\mathrm{K}} n^{4} \longrightarrow \bar{g}_{\mathrm{K}} \frac{Q_{\mathrm{K}}(t)}{N_{\mathrm{K}}}, \quad \bar{g}_{\mathrm{Na}} m^{3} h \longrightarrow \bar{g}_{\mathrm{Na}} \frac{Q_{\mathrm{Na}}(t)}{N_{\mathrm{Na}}},
$$

where $Q_{\mathrm{K}(\mathrm{Na})}$ is the number of fully open and conducting potassium (sodium) channels, $N_{\mathrm{K}(\mathrm{Na})}$ is the total number of potassium (sodium) channels, and $\bar{g}_{\mathrm{K}}$ and $\bar{g}_{\mathrm{Na}}$ have been previously defined. The total number of potassium and sodium channels depends on channel density, $\rho$, and membrane area, $A$, according to $N_{\mathrm{K}}=\rho_{\mathrm{K}} \times A$ and $N_{\mathrm{Na}}=\rho_{\mathrm{Na}} \times A$. Commonly accepted values for channel density in the classic type-II Hodgkin-Huxley parametrization are $\rho_{\mathrm{K}}=18$ and $\rho_{\mathrm{Na}}=60 \mu \mathrm{m}^{-2}$. For simplicity, we use these same values in simulations of both type-I and type-II parametrizations (see Table I). Given the state transition rates specified in Figs. 1 and 2, the stochastic Hodgkin-Huxley Markov-chain model is known to approach the behavior of the deterministic HodgkinHuxley model in the limit of infinite numbers of potassium and sodium channels [19]. Following others [20-22], we use the Markov-chain model as the gold-standard method for implementing channel noise. Extensive numerical simulations of the Markov instantiations of the type-I and type-II models, tested across a range of fixed subthreshold input currents, 
TABLE II. Voltage-dependent gating variable rate constants of the Eq. (1) type-I integrator [15,16] and type-II resonator [18] HodgkinHuxley models explored in this paper. Rate constant units are $\mathrm{ms}^{-1}$ in all cases.

\begin{tabular}{lcc}
\hline \hline Symbol & Integrator & Resonator \\
\hline$\alpha_{n}(V)$ & $-0.032(V+50) /\{\exp [-(V+50) / 5]-1\}$ & $0.01(V+50) /\{1-\exp [-(V+50) / 10]\}$ \\
$\alpha_{m}(V)$ & $-0.32(V+52) /\{\exp [-(V+52) / 4]-1\}$ & $0.1(V+35) /\{1-\exp [-(V+35) / 10]\}$ \\
$\alpha_{h}(V)$ & $0.128 \exp [-(V+48) / 18]$ & $0.07 \exp [-(V+60) / 20]$ \\
$\beta_{n}(V)$ & $0.5 \exp [-(V+55) / 40]$ & $0.125 \exp [-(V+60) / 80]$ \\
$\beta_{m}(V)$ & $0.28(V+25) /\{\exp [(V+25) / 5]-1\}$ & $4 \exp [-(V+60) / 18]$ \\
$\beta_{h}(V)$ & $4 /\{\exp [-(V+25) / 5]+1\}$ & $1 /\{\exp [-(V+30) / 10]+1\}$ \\
\hline \hline
\end{tabular}

are used to establish the canonical behavior of subthreshold transmembrane potential fluctuations, as illustrated in Sec. IV. We then compare the Markov fluctuation responses against that of the approximating channel and linearized SDE models presented next.

\section{B. Channel-based SDE model}

Originally constructed by Fox and Lu [14] via system-size expansion of the master equations describing the potassium and sodium Markov chains given in Figs. 1 and 2, the channel-based SDE models each ion channel as a multivariate stochastic process with appropriately weighted white-noise perturbations. Following Ref. [21], we write this model as

$$
\begin{aligned}
C \frac{d V}{d t}= & I_{\mathrm{DC}}-\bar{g}_{\mathrm{Na}} Y_{31}\left(V-E_{\mathrm{Na}}\right)-\bar{g}_{\mathrm{K}} X_{4}\left(V-E_{\mathrm{K}}\right) \\
& -g_{L}\left(V-E_{L}\right) \\
\frac{d \mathbf{X}}{d t}= & \mathbf{A}_{\mathrm{K}} \mathbf{X}+4 \alpha_{n}(V) X_{0} \mathbf{e}_{\mathbf{1}}+\mathbf{S}_{\mathrm{K}} \mathbf{\Xi}_{\mathrm{K}} \\
\frac{d \mathbf{Y}}{d t}= & \mathbf{A}_{\mathrm{Na}} \mathbf{Y}+3 \alpha_{m}(V) Y_{00} \mathbf{e}_{\mathbf{1}}+\alpha_{h}(V) Y_{00} \mathbf{e}_{\mathbf{4}}+\mathbf{S}_{\mathrm{Na}} \mathbf{\Xi}_{\mathrm{Na}}
\end{aligned}
$$

where $\mathbf{X}=\left[X_{1}, X_{2}, X_{3}, X_{4}\right]^{\mathrm{T}}$ and $\mathbf{Y}=\left[Y_{10}, Y_{20}, Y_{30}, Y_{01}\right.$, $\left.Y_{11}, Y_{21}, Y_{31}\right]^{\mathrm{T}}$ are potassium and sodium channel state vectors where each $X_{i}$ element gives the proportion of potassium channels in the $n_{i}$ state according to Fig. 1, and each $Y_{i j}$ element gives the proportion of sodium channels in the $m_{i} h_{j}$ state according to Fig. 2. In Eq. (3), $\mathbf{A}_{\mathrm{K}(\mathrm{Na})}$ is the drift matrix for the potassium (sodium) channel containing transmembrane potential-dependent channel state transition rates; $\mathbf{S}_{\mathrm{K}(\mathrm{Na})}$ is the matrix square root of the potassium (sodium) channel diffusion matrix, $\mathbf{D}_{\mathrm{K}(\mathrm{Na})}$, containing transmembrane potential-dependent channel state white-noise scalings (i.e., $\mathbf{S}_{\mathrm{K}(\mathrm{Na})}=\sqrt{\left.\mathbf{D}_{\mathrm{K}(\mathrm{Na})}\right)} ; \boldsymbol{\Xi}_{\mathrm{K}(\mathrm{Na})}$ is a column vector containing the potassium (sodium) channel Gaussian white-noise processes

$$
n_{0} \underset{\beta_{n}}{\stackrel{4 \alpha_{n}}{\rightleftarrows}} n_{1} \underset{2 \beta_{n}}{\stackrel{3 \alpha_{n}}{\rightleftarrows}} n_{2} \underset{3 \beta_{n}}{\stackrel{2 \alpha_{n}}{\rightleftarrows}} n_{3} \underset{4 \beta_{n}}{\stackrel{\alpha_{n}}{\rightleftarrows}} n_{4}
$$

FIG. 1. Markov-chain model (kinetic scheme) of the HodgkinHuxley potassium channel with four activation $n$ gates. There are five states in total, each characterized by a different number of open gates, and eight possible state transitions. The only fully open and conducting state is $n_{4}$. State subscripts indicate the number of open $n$ gates and state transition rates are given next to the associated arrow. with zero mean and unit variance; and $\mathbf{e}_{\mathbf{i}}$ is a column vector with a one in the $i^{\text {th }}$ position and zeros elsewhere. Note that this formulation takes advantage of the fact that elements of the vectors $\mathbf{X}$ and $\mathbf{Y}$ describe proportions of channels in the corresponding states such that

$$
\sum_{i=0}^{4} X_{i}=1, \quad \sum_{i=0}^{3} \sum_{j=0}^{1} Y_{i j}=1,
$$

apply and the system can be described in terms of 12 variables rather than 14. Complete expressions for the matrix elements of $\mathbf{A}_{\mathrm{K}(\mathrm{Na})}$ and $\mathbf{D}_{\mathrm{K}(\mathrm{Na})}^{\mathrm{eq}}$ (see below) are given in Appendix B.

While lower-dimensional stochastic differential equations have been proposed to model the underlying dynamics of the Markov-chain description, not all such models have been shown to accurately capture its behavior [20]. Of those models capable of reproducing the Markov-chain dynamics (e.g., see Ref. [23]), we selected the channel-based SDE model because it possesses a clear and direct connection to the Markov chains of Figs. 1 and 2. One disadvantage of this model is that when transmembrane potential is allowed to vary with time, the matrix square root of the diffusion matrices $\mathbf{D}_{\mathrm{K}(\mathrm{Na})}$ must be computed, which is computationally time-consuming and admits the possibility of the $\mathbf{X}$ and $\mathbf{Y}$ state vector elements exceeding their physiologically acceptable bounds: $0 \leqslant X_{i} \leqslant 1$ and $0 \leqslant Y_{i j} \leqslant 1$. To avoid this issue we employ the equilibrium noise approximation wherein, for a given input current, each diffusion matrix is approximated by its value at the steady-state transmembrane potential and we set $\mathbf{D}_{\mathrm{K}(\mathrm{Na})} \approx$ $\mathbf{D}_{\mathrm{K}(\mathrm{Na})}^{\mathrm{eq}}$, and therefore $\mathbf{S}_{\mathrm{K}(\mathrm{Na})} \approx \mathbf{S}_{\mathrm{K}(\mathrm{Na})}^{\text {eq }}$, in Eqs. (3b) and $(3 \mathrm{c})$. In Sec. IV, transmembrane potential fluctuation statistics computed from numerical simulations of this 12-variable SDE model are compared to those of the Markov-chain model to

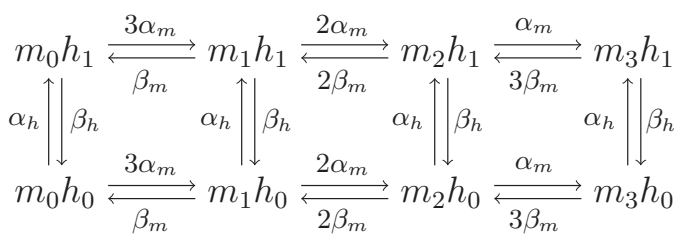

FIG. 2. Markov-chain model (kinetic scheme) of the HodgkinHuxley sodium channel with three activation $m$ gates and one inactivation $h$ gate. There are a total of 8 states, each characterized by a different number of open gates, and 20 possible state transitions. The only fully open and conducting state is $m_{3} h_{1}$. State subscripts indicate the number of open $m$ and $h$ gates and state transition rates are given next to the associated arrow. 
establish its validity in the subthreshold regime. In this way, the nonlinear channel-based SDE serves as a midway point in the progression from the Markov model to the linearized SDE model presented below.

\section{Linear channel-based SDE model}

To aid exploration of critically slowed subthreshold dynamics in the Hodgkin-Huxley model induced by channel noise, we follow Ref. [12] and linearize the channel-based SDE model about the steady-state $\mathbf{Z}^{0}=$ $\left[V^{0}, X_{1}^{0}, X_{2}^{0}, X_{3}^{0}, X_{4}^{0}, Y_{10}^{0}, Y_{20}^{0}, Y_{30}^{0}, Y_{01}^{0}, Y_{11}^{0}, Y_{21}^{0}, Y_{31}^{0}\right]^{\mathrm{T}}$ to form the multivariate Ornstein-Uhlenbeck process

$$
\frac{d}{d t} \mathbf{z}(t)=-\mathbf{A} \mathbf{z}(t)+\sqrt{\mathbf{D}} \mathbf{\Xi}(t)
$$

where $\mathbf{z}(t)=\mathbf{Z}(t)-\mathbf{Z}^{0}$ is a 12-element column vector containing the fluctuation of each variable about its steady-state value, $\mathbf{A}$ and $\mathbf{D}$ are the drift and diffusion matrices of the aggregate system, and $\boldsymbol{\Xi}$ is a column vector containing the 11 independent white-noise perturbations with $\Xi_{1}=0$ (i.e., there is no direct noise perturbation of the transmembrane potential). We apply the equilibrium noise approximation and note that the $\sqrt{\mathbf{D}}$ term in Eq. (5) is given by the matrix $\mathbf{S}$ with block diagonal form $\mathbf{S}=\operatorname{diag}\left(0, \mathbf{S}_{\mathrm{K}}, \mathbf{S}_{\mathrm{Na}}\right)$. The drift matrix, $\mathbf{A}$, is equal to the negative of the Jacobian matrix, $\mathbf{J}$, of Eq. (3) evaluated at the nominated steady state (i.e., $\mathbf{A}=-\mathbf{J}$ ),

$$
\mathbf{J}=\left.\frac{\partial\left(F_{V}, F_{X_{1}}, \ldots, F_{Y_{31}}\right)}{\partial\left(V, X_{1}, \ldots, Y_{31}\right)}\right|_{\mathbf{Z}^{0}} .
$$

Note that there is no simple relationship between the drift matrix $\mathbf{A}$ of Eq. (5) and the $\mathbf{A}_{\mathrm{K}(\mathrm{Na})}$ matrices of Eqs. (3b) and (3c), unlike the direct relationship between $\mathbf{S}$ and $\mathbf{S}_{\mathrm{K}(\mathrm{Na})}$. Stationary solutions to Eq. (5) will exist in the subthreshold regime where each of the 12 eigenvalues, $0>\operatorname{Re}\left(\lambda_{1}\right) \geqslant$ $\operatorname{Re}\left(\lambda_{2}\right) \geqslant \cdots \geqslant \operatorname{Re}\left(\lambda_{12}\right)$, of the Jacobian matrix are negative or have a negative real part. The inverse of the real part of each eigenvalue of $\mathbf{J}$ defines a characteristic time scale for the dynamical development of perturbations in a region close to the steady state. The dominant (i.e., the least negative) eigenvalue, $\lambda_{1}$, determines the slowest of these time scales: $T_{\text {slow }}=1 /\left|\operatorname{Re}\left(\lambda_{1}\right)\right|$.

Following Gardiner [24], stochastic calculus gives the general solution to Eq. (5) in the subthreshold regime as

$$
\mathbf{A} \boldsymbol{\Sigma}+\boldsymbol{\Sigma} \mathbf{A}^{\mathrm{T}}=\mathbf{D}
$$

where $\boldsymbol{\Sigma}$ is the $12 \times 12$ covariance matrix. As detailed in Appendix C, computation of the covariance matrix elements for a given subthreshold stimulus current proceeds by formulating Eq. (7) as a system of linear equations in the unknown matrix elements $\Sigma_{i j}$. The $\Sigma_{11}$ element of the covariance matrix gives the variance, $\operatorname{var}\{\delta v\}$, of transmembrane potential deviations, $\delta v(t)=V(t)-V^{0}$, about the $V^{0}$ steady state at each input current. To capture the essential components of dynamical slowing - growth in amplitude simultaneous with decay in frequency of transmembrane potential fluctuationswe consider the time-correlation matrix and its Fourier transform, the spectrum matrix. The time-correlation matrix, $\mathbf{C}(\tau)$, with symmetry property $\mathbf{C}(-\tau)=[\mathbf{C}(\tau)]^{\mathrm{T}}$, is given by

$$
\mathbf{C}(\tau)=e^{-\mathbf{A} \tau} \boldsymbol{\Sigma}, \quad \tau \geqslant 0
$$

where $\tau$ is the time lag. Likewise, the spectrum matrix, $\mathbf{S}(\omega)$, is given by [24]

$$
\mathbf{S}(\omega)=\frac{1}{2 \pi} \int_{-\infty}^{\infty} e^{-i \omega \tau} \mathbf{C}(\tau) d \tau,
$$

where $\omega$ is frequency. Note that the analysis presented here is only applicable in the subthreshold regime: On crossing threshold at least one eigenvalue of the Jacobian matrix will satisfy $\operatorname{Re}\left(\lambda_{i}\right)>0$ and a stationary solution to Eq. (5) will no longer exist.

Predictions derived from Eqs. (7), (8), and (9) are compared to transmembrane potential fluctuation statistics obtained from simulated Markov-chain and full channel-based SDE stochastic trajectories. When combined with eigenvalue analysis of the Jacobian matrix, the linearized system is used to provide insight into the observed dynamical behavior on approach to threshold.

\section{NUMERICAL SIMULATIONS}

The three models considered in this study were implemented in MATLAB, versions R2013b and/or R2014a. All computations were performed using double-precision (64-bit) floating-point arithmetic with the exception of the linear channel-based SDE model for which a combination of the symbolic and variable-precision arithmetic capabilities of MATLAB's Symbolic Math Toolbox (version 6.0) were also used. For the Markov-chain ion-channel and channel-based SDE models, we simulated stochastic transmembrane potential trajectories for both the type-I integrator and type-II resonator parametrizations (see Table I) as a function of DC input current density, $I_{\mathrm{DC}}$, for fixed membrane area, $A$. For each parametrization, the subthreshold regime is defined by $I_{\mathrm{DC}}<$ $I_{\mathrm{DC}}^{\text {crit }}$, where $I_{\mathrm{DC}}^{\text {crit }}$ is the critical current density for spike generation in the Eq. (1) deterministic Hodgkin-Huxley model. Because the stochastic nature of these models can result in subthreshold action potential generation, membrane areas in the 3000- to $4000-\mu \mathrm{m}^{2}$ range were utilized in order to attenuate random spiking activity and allow for sufficiently long epochs of quiescent spike-free activity for analysis. Spike detection in simulated traces was accomplished via an algorithm based on the transmembrane potential crossing a threshold value (typically chosen to be $0 \mathrm{mV}$ ). For each detected spike, both the prior $20 \mathrm{~ms}$ and subsequent $40 \mathrm{~ms}$ of simulated time were excluded from analysis. Variation of these exclusion times did not significantly influence the fluctuation statistics computed from the spike-free epochs. Simulation times of at least $10 \mathrm{~s}$ were used at each input current density. The initial conditions for all simulations corresponded to the input current-density-dependent deterministic equilibrium state.

The subthreshold Markov-chain ion-channel model, the accepted standard for implementation of channel noise, was numerically simulated using the Gillespie stochastic simulation algorithm (SSA) [25] as implemented by Chow and White [26]. Briefly, at each iteration of the algorithm, two uniformly distributed pseudorandom numbers are used to determine (i) the time until the next transition and (ii) the 
(a) Markov Integrator: Area $=3200 \mu \mathrm{m}^{2}$

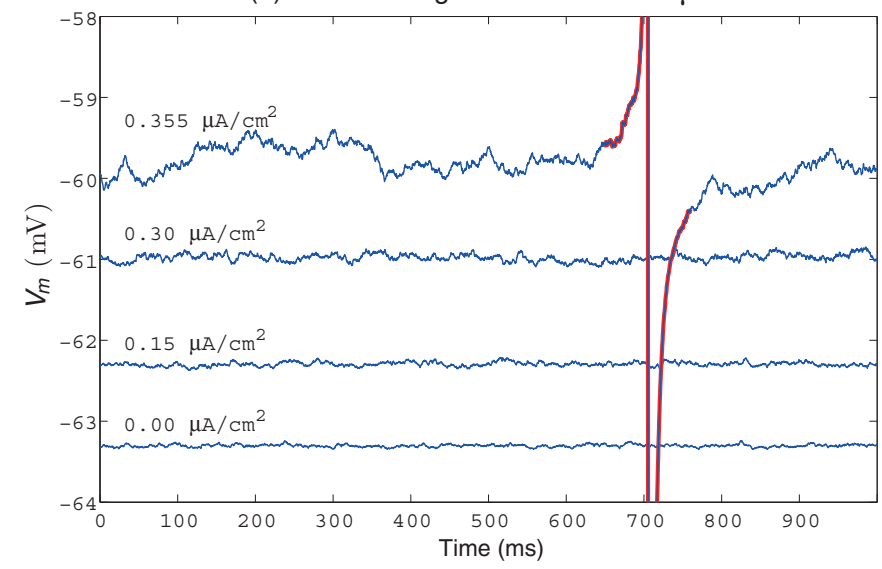

(b) Markov Resonator: Area $=4000 \mu \mathrm{m}^{2}$

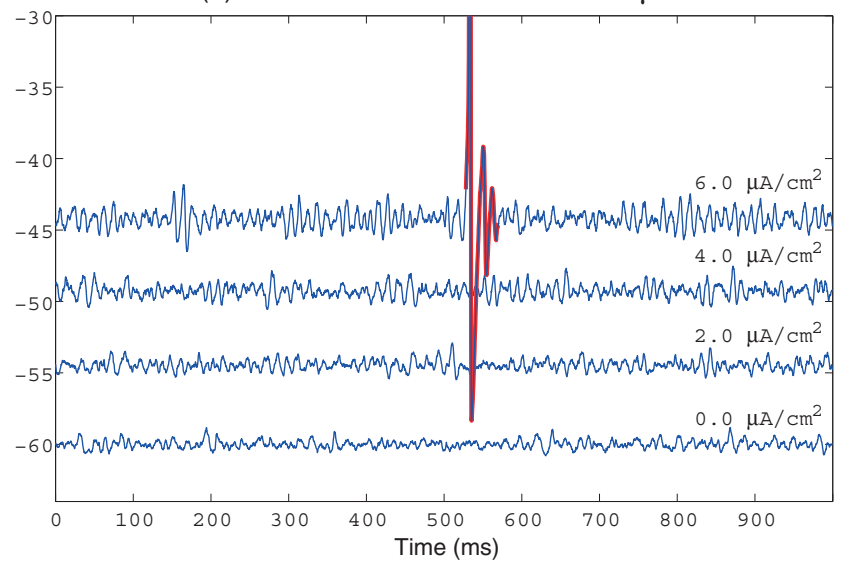

FIG. 3. (Color online) Markov-chain stochastic simulations of a Hodgkin-Huxley neuron configured as (a) a type-I integrator and (b) a type-II resonator for a sequence of four distinct subthreshold current-density values $I_{\mathrm{DC}}\left(\right.$ in $\mu \mathrm{A} / \mathrm{cm}^{2}$ ) for each neuron type. See Table I for parameter values and Figs. 1 and 2 for Markov-chain kinetics. Blue (gray) traces show membrane potential, $V_{m}$, fluctuations induced by stochastic channel-state transitions; fluctuations become larger and more spectrally colored as spiking threshold is approached from below. In both cases, the top trace captures a single spiking event, shown in thick red (thick gray), that has been cropped to highlight baseline fluctuations. For each run, all 14 state variables (five $\mathrm{K}^{+}$channel states, eight $\mathrm{Na}^{+}$channel states, and membrane potential) were initialized at the deterministic steady state and then iterated using the Chow and White [26] implementation of the Gillespie stochastic simulation algorithm (SSA) [25] and sampled at deterministic time intervals $\Delta t=0.005 \mathrm{~ms}$ for $10 \mathrm{~s}$ of simulated time; $1 \mathrm{~s}$ extracts are shown here. Traces in (b) have been offset vertically to reduce overlap (offset in $\mathrm{mV}=[0,4,8,12]$ counting from bottom to top). These Markov simulations are computationally demanding (MATLAB run times $\sim 64 \mathrm{~h}$ per $10 \mathrm{~s}$ trace on an Intel Xeon $2.80-\mathrm{GHz} \mathrm{CPU}$ ).

(a) Integrator Histograms: Simulation vs Theory

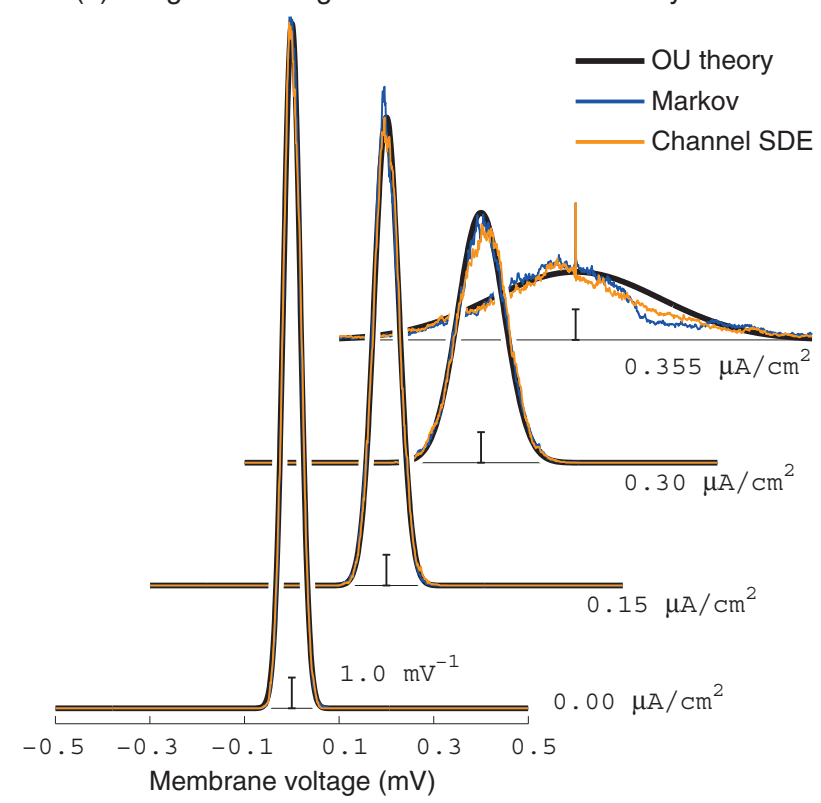

(b) Resonator Histograms: Simulation vs Theory

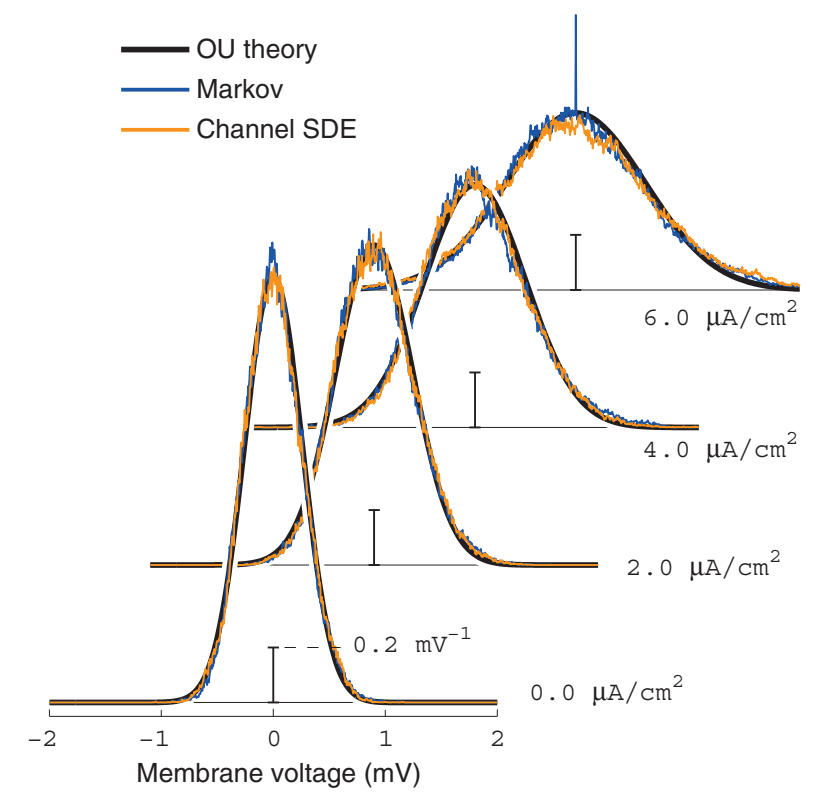

FIG. 4. (Color online) Distribution densities for subthreshold voltage fluctuations in (a) type-I integrator and (b) type-II resonator HodgkinHuxley neurons, comparing Markov [blue (gray)] and SDE [orange (light gray)] numerical simulations with linear Ornstein-Uhlenbeck (OU) theory (thick black). The Markov and SDE probability densities are estimated from their respective $V_{m}$ time series by computing transmembrane potential histograms and then dividing by $N \Delta V$ with $N=2001$ being the number of bins and $\Delta V$ the bin width. For each $I_{\mathrm{DC}}$ simulation, the thick black reference curve is the OU prediction that the subthreshold fluctuations will be Gaussian distributed with zero mean. Vertical scale markers carry units of $\mathrm{mV}^{-1}$ and the total area under each curve is a dimensionless unity. Distributions from numerical simulations accurately follow theoretical prediction, even at the highest currents that result in occasional action potential generation. The broadening of the distributions corresponds to increased fluctuation amplitude as instability threshold is approached from below. (The "thorns" riding on the top of the maximum-current Markov and SDE histograms are a signature artifact generated by the zeroing of action-potential events detected in the time series.) 
(a) Integrator Correlations: Simulation vs Theory

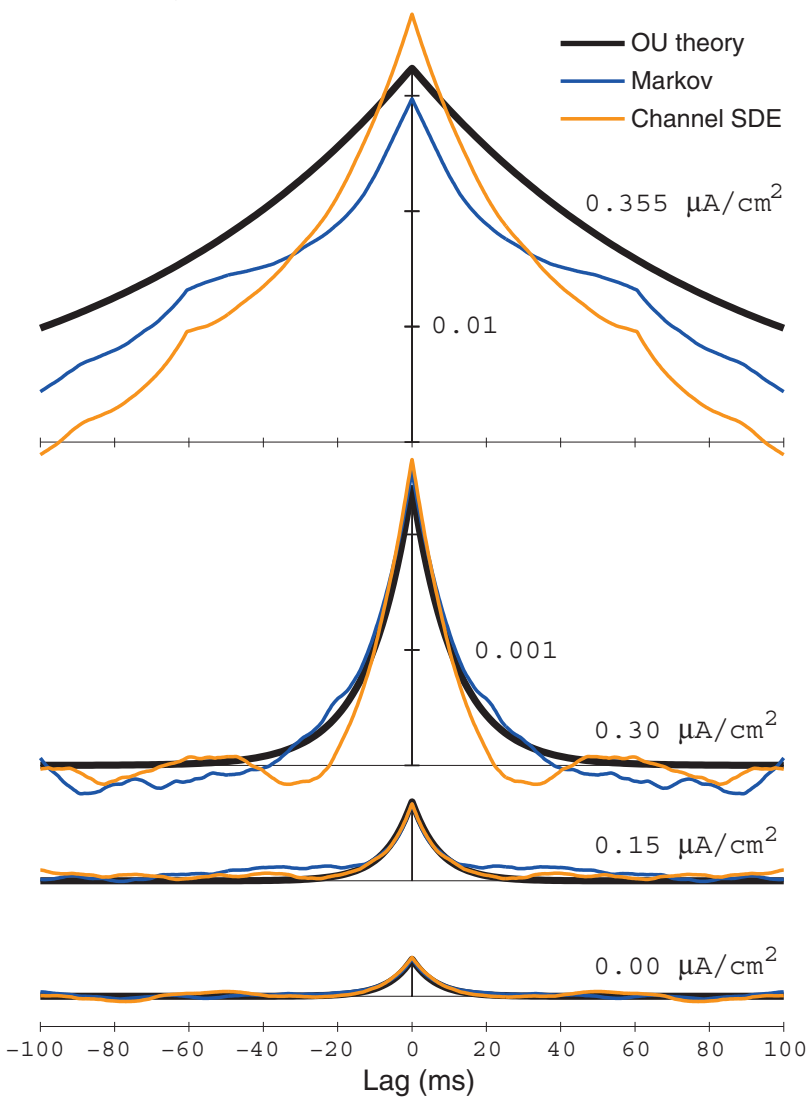

(b) Resonator Correlations: Simulation vs Theory
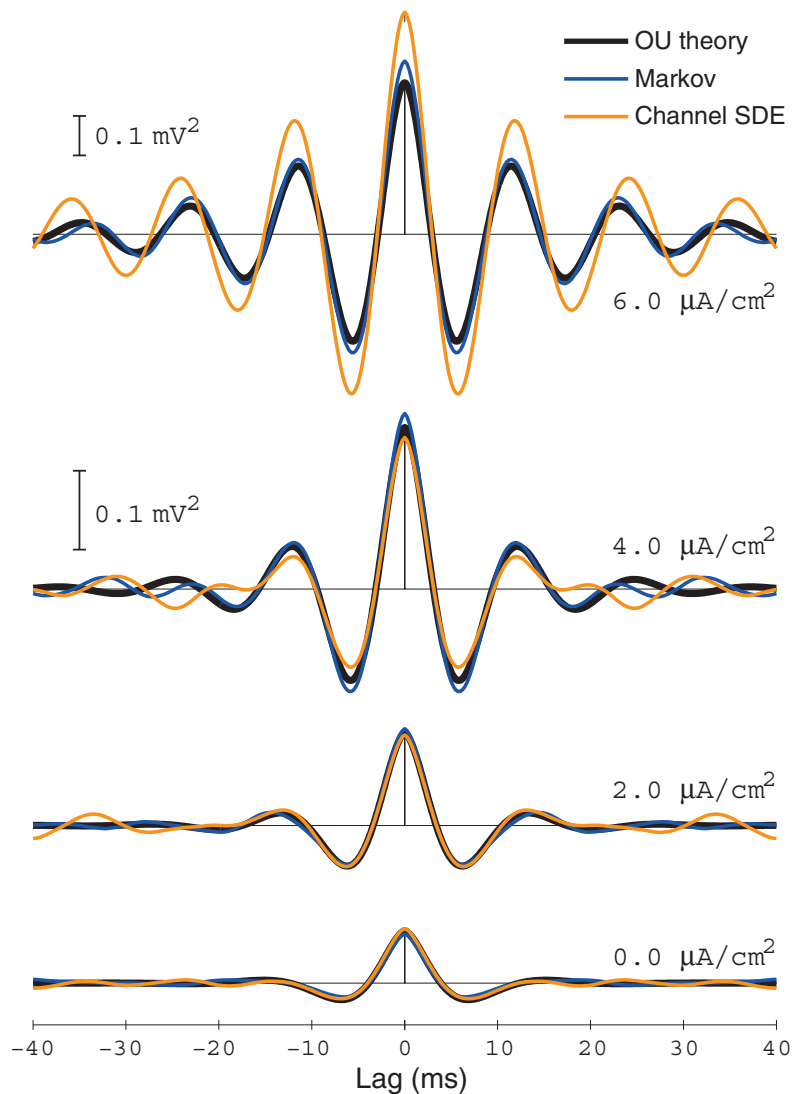

FIG. 5. (Color online) Autocorrelation functions for subthreshold transmembrane potential fluctuations in the (a) type-I integrator and (b) type-II resonator Hodgkin-Huxley neurons, comparing Markov [blue (gray)] and SDE [orange (light gray)] numerical simulations with linear Ornstein-Uhlenbeck (OU) theory (thick black). For both neuron types, there is a dramatic surge in variance (zero-lag autocorrelation) and temporal persistence (time for the decay-envelope to drop to 1/e of the zero-lag maximum) as spiking threshold is approached from below. We note the excellent agreement between the linear OU prediction and the Markov and SDE simulations; although the SDE numerics run about $200 \times$ faster than the Markov SSA codes, both algorithms produce output that is statistically indistinguishable. In (a), the bottom three traces are calibrated to the $0.001-\mathrm{mV}^{2}$ vertical scale marker, while the top trace has been rescaled to a $0.01-\mathrm{mV}^{2}$ interval. The Markov correlations were computed from the full 10-s Markov recordings (see Fig. 3) by averaging autocorrelations from five 2000-ms nonoverlapping segments; the SDE traces were computed from a corresponding set of SDE simulation runs (not shown); the OU curves are predictions from OU theory [see Eqs. (7) and (8)]. Spike events occurred occasionally (about 12 spikes per $10 \mathrm{~s}$ ) at the highest level of $I_{\mathrm{DC}}$ drive for the integrator neuron; these events were detected and suppressed by setting the transmembrane potential fluctuation to zero for the spike duration.

specific ion-channel transition that occurs (see Appendix A for further discussion). Although we sampled the generated time series at deterministic time intervals of $\Delta t=0.005 \mathrm{~ms}$, the internal time step of the algorithm is inherently variable and many state transitions may occur in the period of time between two deterministic samplings. This implementation of Gillespie's SSA tracks the total number of channels in each of the five possible sodium channel states and eight possible potassium channel states and is therefore more efficient than algorithms which track the states of each individual potassium and sodium channel as done in Ref. [27]. Nevertheless, the Chow and White implementation is still computationally very expensive, requiring $\sim 6 \mathrm{~h}$ of run time per second of simulation time for large membrane areas $\left(A \sim 4000 \mu \mathrm{m}^{2}\right)$. Due to the inverse relationship between the total number of ion channels $N=N_{\mathrm{K}}+N_{\mathrm{Na}}$ and the time between transitions in Gillespie's SSA, the computational demand of the algorithm scales linearly with membrane area $\left[A=N_{\mathrm{K}(\mathrm{Na})} / \rho_{\mathrm{K}(\mathrm{Na})}\right]$. Examples of Markov-chain ion- channel model transmembrane potential tracings are given in Fig. 3.

The channel-based SDE model was numerically integrated in the subthreshold regime using the Euler-Maruyama algorithm [28] with a deterministic time step, $\Delta t=0.005 \mathrm{~ms}$, equal to that used for sampling the Markov-chain model simulations. As described earlier, the equilibrium noise approximation was applied in all simulations. In contrast to the full channel-based SDE model, the linearized version was not simulated to produce transmembrane potential traces. Rather, the Eq. (7) solution of the linear channel-based SDE model was solved directly for the covariance matrix elements from which all investigated quantities were computed (see Appendix $\mathrm{C}$ for details).

\section{RESULTS}

One of the striking features of the sample Markov-chain membrane voltage tracings given in Fig. 3 is the noticeable 
(a) Integrator Spectra: Simulation vs Theory
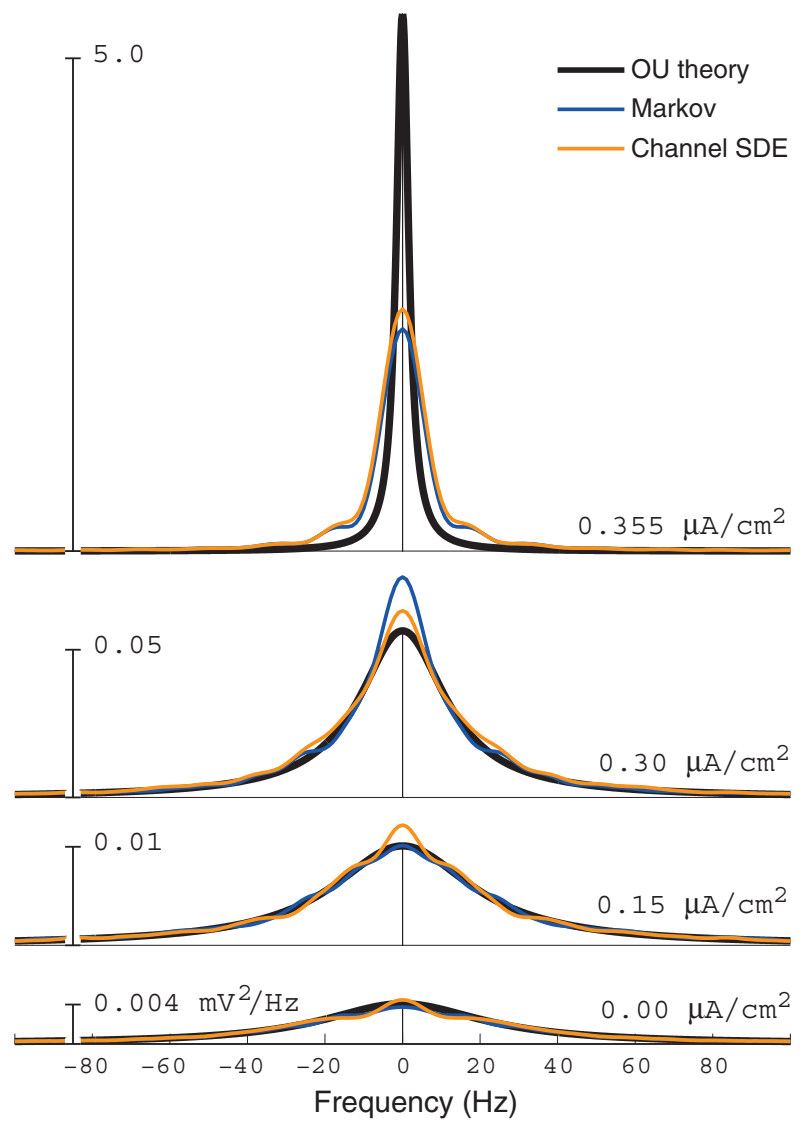

(b) Resonator Spectra: Simulation vs Theory

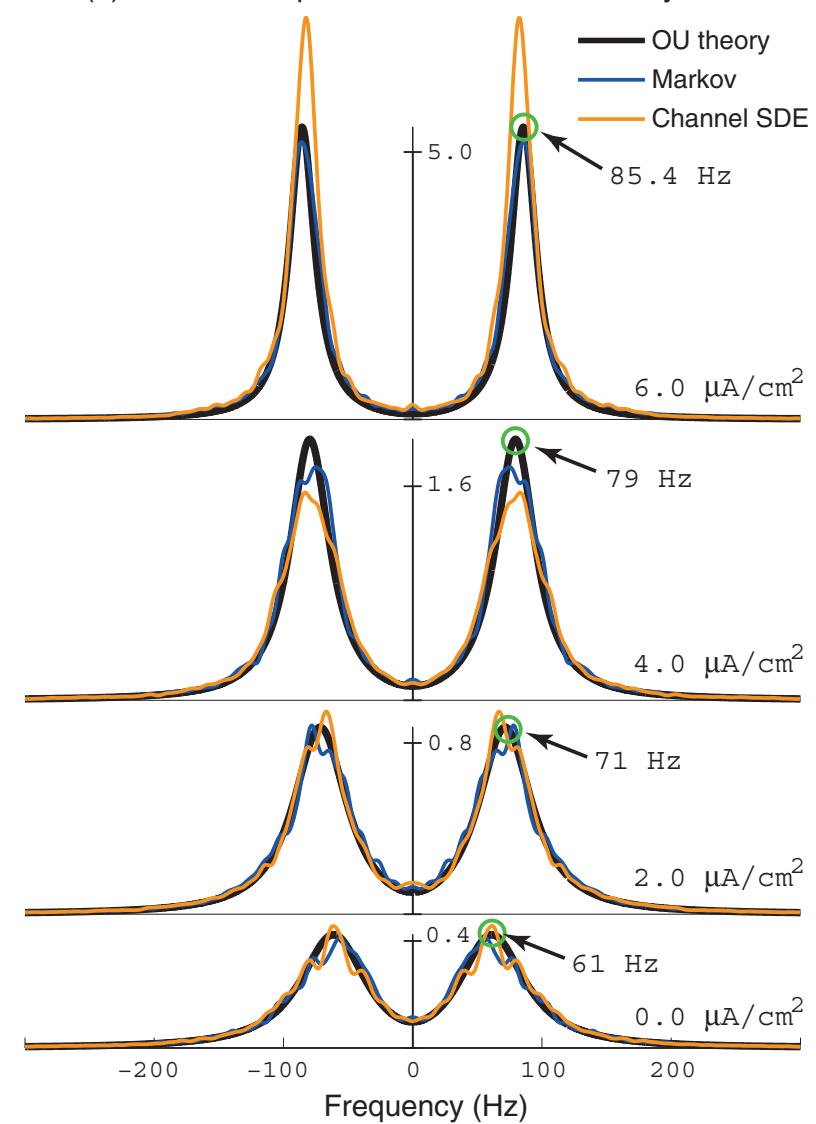

FIG. 6. (Color online) Power spectral densities for subthreshold voltage fluctuations in the (a) type-I integrator and (b) type-II resonator Hodgkin-Huxley neurons, comparing Markov [blue (gray)] and SDE [orange (light gray)] numerical simulations with linear Ornstein-Uhlenbeck (OU) theory (thick black). These curves are the Fourier-domain equivalent of the autocorrelation functions plotted in Fig. 5, but the Markov and SDE spectra were computed directly from Markov (Fig. 3) and SDE numerical simulations (not shown) via the MATLAB spectrogram function (10 $000 \mathrm{~ms}$ recordings at 200-kHz sample rate, $100 \mathrm{~ms}$ epochs with $50 \%$ overlap, and top-hat window); the OU theoretical spectra are linear predictions from Eqs. (7) and (9). Vertical scale markers carry units of $\mathrm{mV}^{2} / \mathrm{Hz}$. Total area under each curve estimates the $V_{m}$ time-series fluctuation variance (i.e., zero-lag correlation peak in Fig. 5). Agreement between numerically simulated and theoretical spectra is generally excellent, except for the topmost trace of (a) where the 100-ms epoch lengths are too short to adequately capture the energy at zero frequency. (Much longer epochs can retrieve a more accurate low-frequency peak but at the cost of degraded signal-to-noise from the diminished number of spectral samples available to be averaged.)

increase in the magnitude of subthreshold excursions from equilibrium as the threshold current density, $I_{\mathrm{DC}}^{\text {crit }}$, is approached from below. For each of the three models considered here, these fluctuations are illustrated in Fig. 4 for both the type-I integrator and type-II resonator parametrizations and were further quantified by computing the autocorrelation functions and their associated power spectral densities given, respectively, in Figs. 5 and 6. While both the type-I integrator and type-II resonator autocorrelation functions of Fig. 5 are characterized by increasing zero-lag magnitudes and diminishing damping as $I_{\mathrm{DC}} \rightarrow I_{\mathrm{DC}}^{\text {crit }}$, there is a clear distinction between the two sets of curves: Unlike the type-I integrator curves which decrease monotonically with increasing time lag, the typeII resonator curves show damped oscillations. The power spectral densities given in Fig. 6 provide complementary illustrations of the autocorrelation divergences associated with approach to spiking threshold from below. For the type-I integrator in Fig. 6(a), there is increasing spectral coloration of transmembrane potential fluctuations sharply peaked around $\omega=0$ as the input current density approaches threshold. This behavior contrasts with the type-II resonator in Fig. 6(b) where we see a resonance peak $\left(\sim 61 \mathrm{~Hz}\right.$ at $I_{\mathrm{DC}}=0, \sim 85 \mathrm{~Hz}$ at $6.0 \mu \mathrm{A} / \mathrm{cm}^{2}$ ) whose magnitude diverges as the resonance becomes more narrowly focused for $I_{\mathrm{DC}} \rightarrow I_{\mathrm{DC}}^{\text {crit }}$.

The prolongation in decay time illustrated in the Fig. 5 autocorrelation functions is further quantified in Fig. 7 which gives the dependence of the $1 / e$ decay times as a function of the dimensionless parameter $\epsilon \equiv\left(I_{\mathrm{DC}}^{\text {crit }}-I_{\mathrm{DC}}\right) / I_{\mathrm{DC}}^{\text {crit }}$, which quantifies distance from spiking threshold. This figure also gives the dependence of the characteristic time scales associated with each of the three least-negative eigenvalues of the Ornstein-Uhlenbeck linearization of the channel-based SDE model. It is clear that the characteristic time scale $T_{\text {slow }}=$ $1 /\left|\operatorname{Re}\left(\lambda_{1}\right)\right|$ associated with the dominant eigenvalue is in excellent agreement with both the Markov-chain ion-channel and full channel-based SDE model results. Also included in Fig. 7 are the predicted $\tau \propto \epsilon^{-v}$ power laws, where $v \approx 0.5$ for the saddle-node bifurcation chracteristic of type-I behavior 
(a) Integrator: Correlation time vs $\epsilon$

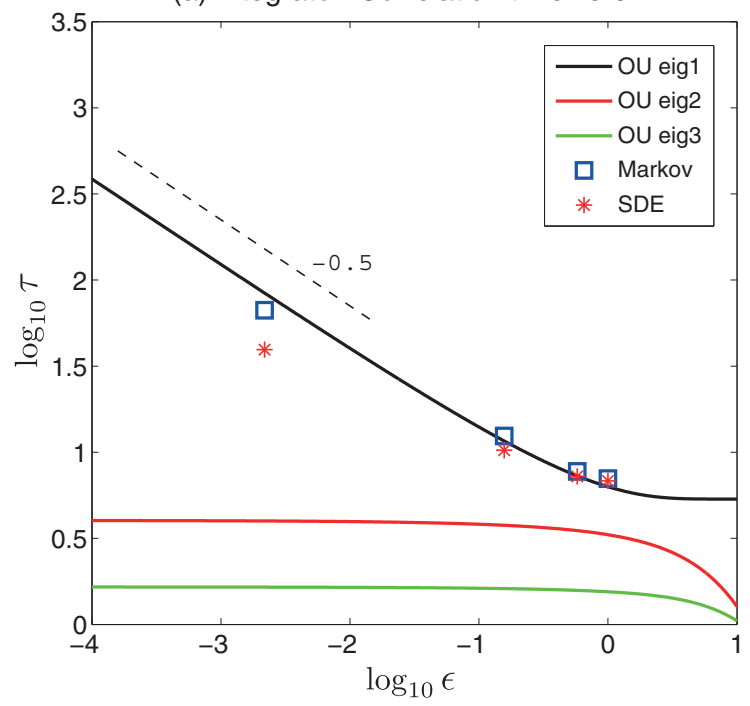

(b) Resonator: Correlation time vs $\epsilon$



FIG. 7. (Color online) Divergence of correlation decay times as a function of proximity to instability threshold in the (a) type-I integrator and (b) type-II resonator Hodgkin-Huxley neurons. Curves show characteristic times $\tau_{i}=1 / \alpha_{i}$ (in ms), with $i=1,2,3$ and $\alpha \equiv-\operatorname{Re}($ eig) being the decay rate predicted by the first 3 (of 12) Ornstein-Uhlenbeck (OU) eigenvalues. The Markov and SDE correlation times are measured as the time required for the autocorrelation functions of their $V_{m}$ fluctuations to decay to 1/e of the zero-lag peak; for (b), we revise this to measure the time for the oscillations envelope to decay to $1 / e$. Evidently these correlation times follow power-law growth trends with (a) $\tau \sim \epsilon^{-0.5}$ for integrator and (b) $\tau \sim \epsilon^{-1}$ for resonator, where $\epsilon \equiv\left(I_{\mathrm{DC}}^{\text {crit }}-I_{\mathrm{DC}}\right) / I_{\mathrm{DC}}^{\text {crit }}$ (see Table I for $I_{\mathrm{DC}}^{\text {crit }}$ values) measures proximity to the instability point as determined by a sign-change in the real part of the dominant eigenvalue in the deterministic equations.

and $v \approx 1$ for the subcritical Andronov-Hopf bifurcation characteristic of type-II behavior.

\section{DISCUSSION}

Using Markov-chain models to simulate the influence of noisy transmembrane ion-channel activity on the dynamics of subthreshold type-I and type-II Hodgkin-Huxley neurons, we have investigated the fluctuation statistics of excitable membranes as the threshold for spike generation is approached from below. We find that close approach to this behavioral transition is associated with signatures of criticality including divergent correlation times and spectral focusing. Indeed, Freeman [29] has characterized the behavior of neurons on close approach to threshold by stating that, "As threshold is approached, a nonlinear domain is encountered in which local responses occur that are greater than expected by proportionality, yet are still below threshold for a propagated action potential. In this domain an excitatory input not only produces depolarization; it sensitizes the neuron to further excitation." The progression of models presented here demonstrate that fluctuations produced by ion-channel noise are sufficient to account for this behavior. Furthermore, because ion-channel noise is an intrinsic feature of neurons in vivo, this behavior is likely an essential component of biologically realistic neuronal dynamics in the subthreshold regime.

From the deterministic point of view, action potential generation is an inherently nonlinear dynamical phenomenon governed by the bifurcation structure of the underlying equations-saddle-node for type-I neurons and subcritical Andronov-Hopf for type-II neurons-on approach to threshold. Close to the subthreshold equilibrium state, the development of these deterministic dynamics is well described by the eigenvalue structure of the corresponding linearized system and a clear connection between the dynamics and the eigenvalues exists. However, this connection is obscured when high-dimensional Markov-chains are used to model finite numbers of ion channels undergoing noisy state-transitions which can result in the generation of highly nonlinear action potentials. To clarify this relationship, we have approximated the Markov-chain model with a channel-based SDE and demonstrated close agreement in their respective fluctuation statistics. Linearization of the full SDE model then allowed for semianalytic treatment and eigenvalue analysis. Despite these SDE models representing successive approximations to the full stochastic dynamics, they are able to adequately account for the observed behavior in simulated subthreshold Markov transmembrane potential tracings. While perhaps most surprising for the linear SDE, this agreement is also notable for the full channel-based SDE given our use of the equilibrium noise approximation. In their thorough voltage-clamp investigation of SDE approximations of ion-channel noise in the classic resonator Hodgkin-Huxley model, Goldwyn et al. [21] also found that the equilibrium noise approximation performed well but questioned its validity when the transmembrane potential is not voltage clamped. Although our investigation is confined to the subthreshold regime, and does not probe suprathreshold behavior such as interspike interval statistics, these results provide additional evidence as to the acceptability of this computationally useful approximation.

To further solidify the relationship between the Markovchain and deterministic linear dynamics, Fig. 8 plots the base-10 logarithm of the transmembrane potential variance as a function of the dimensionless parameter $\epsilon=\left(I_{\mathrm{DC}}^{\text {crit }}-I_{\mathrm{DC}}\right) / I_{\mathrm{DC}}^{\text {crit }}$ 
(a) Integrator Variance vs $\epsilon$

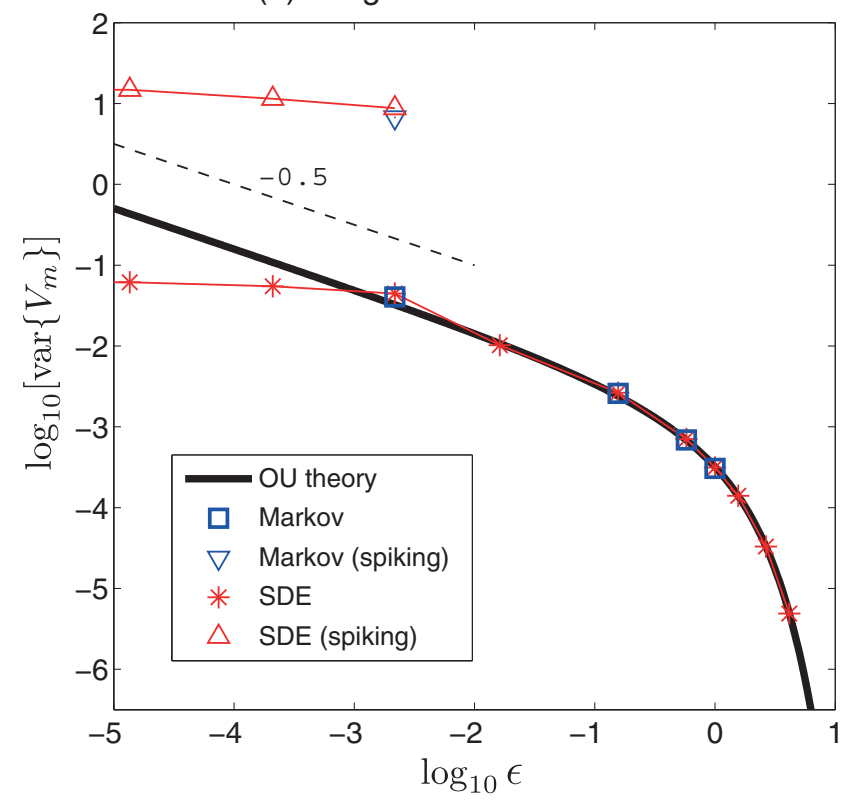

(b) Resonator Variance vs $\epsilon$

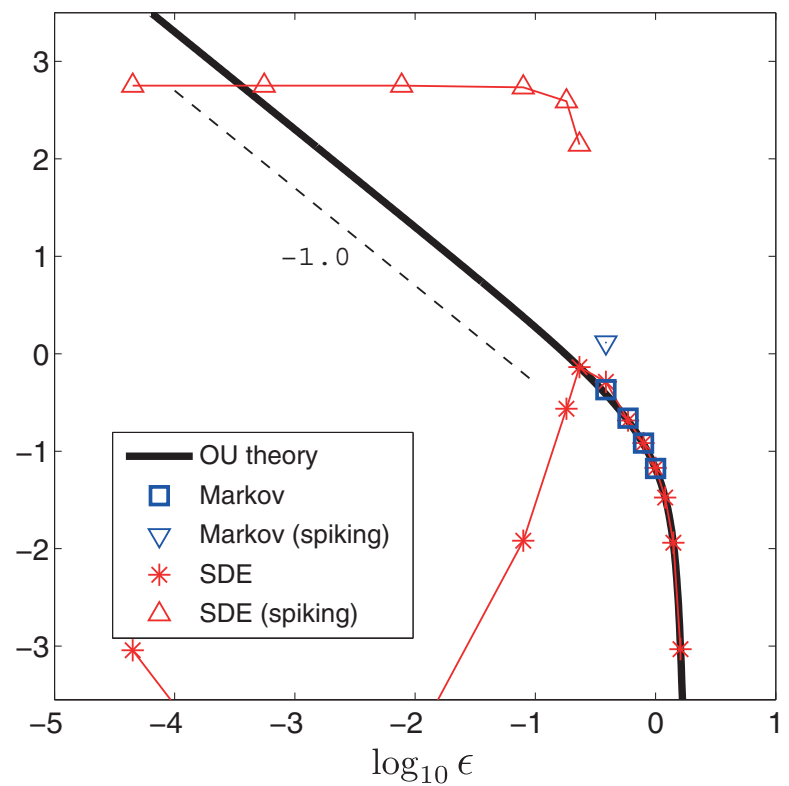

FIG. 8. (Color online) Testing for power-law growth of membrane-voltage fluctuation variance as a function of proximity to instability threshold in (a) type-I integrator and (b) type-II resonator Hodgkin-Huxley neurons. Ornstein-Uhlenbeck (OU) theory predicts that voltage fluctuation power will scale asymptotically as (a) $\epsilon^{-0.5}$ for integrator and (b) $\epsilon^{-1}$ for resonator, where $\epsilon \equiv\left(I_{\mathrm{DC}}^{\text {crit }}-I_{\mathrm{DC}}\right) / I_{\mathrm{DC}}^{\text {crit }}$ is a dimensionless measure of the distance from the critical point. Squares (Markov) correspond to the four sets of currents shown in previous figures, and asterisks (channel-SDE) show spike-suppressed fluctuation variances for stochastic simulations over a much wider range of $I_{\mathrm{DC}}$ values; down-triangles (Markov) and up-triangles (SDE) show fluctuation power when spikes are not suppressed in the variance calculations. The collapse in spike-suppressed fluctuation power for $\epsilon \lesssim 10^{-0.6}$ in (b) arises because resonator spikes tend to occur in bursts, leading to prolonged intervals of zeroed activity, whereas in the integrator (a), intermittent spikes remain brief, solitary events. Prior to emergence of spikes, both Markov and SDE simulations follow OU predictions closely. (Note that the region of concordance would extend further to the left if the membrane area-and hence the number of $\mathrm{Na}$ and $\mathrm{K}$ channels-were increased.)

which quantifies distance from threshold. As expected, the linearized SDE model obeys the deterministically anticipated $\epsilon^{-v}$ scalings as $\epsilon \rightarrow 0$ with $\nu=0.5$ for type-I behavior and $v=1$ for type-II behavior. While broadly consistent behavior is obtained for both the Markov and full SDE models, on close approach to threshold the observed scalings diverge from these predictions. Near the quiescent-to-spiking transition, the emergence of action potentials interrupts the time development of these purely subthreshold predictions such that the critically slowed dynamics contributes to its own annihilation via promoting excursions away from equilibrium. Note that because the rate of generation of spontaneous action potentials decreases as the area of the modelled membrane increases [26], the critically slowed subthreshold dynamics discussed here will be more amenable to observation and may function as, or contribute to, a nonspecific organizing mechanism that is self-reinforcing as the number of ion channels increases.

Utilizing the reduced dimensionality Wilson model [30], previous work on the subthreshold dynamics of point-neuron models by Steyn-Ross et al. [12] resulted in findings consistent with those reported here: divergence of the slow time scale, divergence of fluctuation variance, and focusing of spectral content as threshold is approached from below. One significant difference with the current report is the method used to model biological noise. In Ref. [12], Gaussian white noise added to the transmembrane potential variable was used as a nonspecific method to capture the consequences of the noisy biological environment in which neurons must function. We find this method of accounting for biological reality to be unnecessary; the inherent noisiness of ion-channel state transitions as captured in Markov-chain models is sufficient to account for the critically slowed dynamics observed by Freeman [29] on approach to spiking threshold. While not a substitute for synaptic noise, using ion channels as the noise source via Markov models eliminates the need to select appropriate noise magnitudes in stochastic differential equation formulations. Further investigations of critical slowing-down in computational models incorporating both ion-channel noise and realistic synaptic activity (e.g., see Ref. [31]) should provide additional insight into the range of behaviors described here.

Note that the mathematical form of the divergence in correlation time $T_{\text {slow }}$ for microscopic subthreshold fluctuations in type-I (integrator) neurons (reported here and in Ref. [12]) is preserved as we cross the $I_{\mathrm{DC}}^{\text {crit }}$ threshold to generate macroscopic, highly nonlinear spiking events of period $T$. In both cases, these characteristic response times obey a square-root scaling law: $T \sim 1 /\left|I_{\mathrm{DC}}^{\text {crit }}-I_{\mathrm{DC}}\right|^{0.5}$. [The latter asymptotic spiking behavior in integrator neurons is well established (e.g., see Ref. [9]).] Thus an infinitely slowed noise-induced fluctuation immediately below threshold emerges as an infinite-period (i.e., zero frequency) spike sequence immediately above threshold. This provides an 
unexpected but intuitive continuity across threshold with the type-I fluctuation spectrum of Fig. 6(a) predicting resonance at zero frequency. Although this square-root temporal scaling is a characteristic of the saddle-node bifurcation of fixed points associated with type-I neurons [9], it can also be observed in the relaxation dynamics of type-II neurons. In Ref. [32], Roa et al. investigated the response of several deterministic resonator neuron types (Hodgkin-Huxley, Morris-Lecar, and Fitzhugh-Nagumo) to a step increase in drive current $I$ from zero to a level just below the critical current $I_{c}$ for the saddlenode bifurcation into stable and unstable limit cycles, with $I \lesssim$ $I_{c}<I_{H}$, where $I_{H}$ is the Andronov-Hopf bifurcation point. Numerical simulations demonstrated that the relaxation time $\tau$ for passage through the ghost of the saddle-node bifurcation back to steady state obeyed a power-law scaling with exponent 0.5: $\tau \sim 1 /\left(I_{c}-I\right)^{0.5}$. In contrast to this investigation, the present work places the type-II Hodgkin-Huxley neuron near the Andronov-Hopf bifurcation with a constant current $I \lesssim I_{H}$ and then perturbs the neuron with low-intensity noise and finds spectrally colored fluctuations whose correlation time obeys a scaling law $T_{\text {slow }} \sim 1 /\left(I_{H}-I\right)^{1.0}$ [see Fig. 7(b)]. Thus we conclude that type-II models can exhibit multiple near-threshold scaling behaviors, depending on the region of phase space under consideration.

In this paper we have described the effects of ion-channel noise on subthreshold point neurons via direct simulation of Markov-chain models describing the state transitions of the underlying transmembrane proteins. As a mechanism to promote exploration of the subthreshold phase space we suggest, in line with Ref. [12], that the observed dynamical signatures of critical slowing-down may have biophysical significance. Having been experimentally demonstrated in the squid giant axon preparation [13], we anticipate that observation of critical slowing-down in the neurophysiological laboratory may be possible under appropriate conditions (see Ref. [33] for a recent experimental demonstration). When considering interneuron communication-a process traditionally thought to be constrained to suprathreshold actions-divergence of subthreshold voltage fluctuations just prior to spike initiation may have functional significance given the presence of gap electrical junctions that can couple one neuron to another. While this is presently purely conjecture, the robust effects of noise in nonlinear dynamical systems together with the ubiquity of noise in biological systems may favor this perspective. However, given the high levels of background synaptic activity to which in vivo neurons are exposed, the statistical signatures of critical slowing due to ion-channel fluctuations outlined here may be obscured because their observation appears to require close approach to threshold.

\section{APPENDIX A: STOCHASTIC SIMULATION ALGORITHM}

Gillespie's SSA [25] represents the canonical method for including channel noise in model neurons. Interestingly, there is a subtle ambiguity in the SSA as implemented by Chow and White [26] regarding the ordering of the transmembrane potential integration step and the state-update step. As described in multiple sources (e.g., see Refs. [21] and [22]), once the time to the next transition, $\Delta t_{\text {transition}}$, is computed from the first uniformly distributed pseudorandom number, the ordinary differential equation describing the transmembrane potential's time dependence [see Eqs. (1a) and (2)] is integrated to give the transmembrane potential at the new time (i.e., $t_{\text {new }}=$ $\left.t_{\text {old }}+\Delta t_{\text {transition }}\right)$. This step is followed by determination of the state transition that occurs at $t_{\text {new }}$ from (i) a second uniformly distributed pseudorandom number and (ii) the 28 transmembrane potential-dependent state-transition rates using the transmembrane potential from the previous time, $t_{\text {old }}$. An alternative and logically consistent approach would be to determine the state transition that occurs at the new time by using the transmembrane potential at $t_{\text {new }}$ rather than that at the previous time $t_{\text {old }}$. However, because this alternate method requires an additional calculation of the 28 state-transition rates per time step, there is added computational cost. We have implemented both of these algorithmic choices-using the same sequence of pseudorandom numbers and therefore having the same sequence of transition times-and find that, following a short lag time, the results diverge but are statistically indistinguishable. This behavior is as expected: as the total number of channels increases, the perturbation to the transmembrane potential induced by a single state transition is expected to diminish.

As pointed out by an anonymous referee, the ambiguity discussed above is symptomatic of the "piecewise constant propensity approximation" used by Chow and White [26] in their implementation of the Gillespie algorithm. An exact version of Gillespie's algorithm, presented in a computational neuroscience context, is given by Anderson et al. [34] and references therein. This exact algorithm accounts for the time dependence of state-transition propensities due to the continuously changing membrane voltage between discrete channel transitions. As stated by Anderson et al. [34], "[The] piecewise constant propensity approximation is analogous, in a sense, to the forward Euler method for the numerical solution of ordinary differential equations." While the approximate and exact versions of the Gillespie algorithm are not expected to produce identical sample paths when driven by identical streams of uniform random numbers, paths with similar statistical properties can be anticipated for sufficiently small transition times [34].

\section{APPENDIX B: CHANNEL-BASED SDE MODEL [21]}

Implementation of the channel-based SDE model requires knowledge of the Eqs. (3b) and (3c) drift and diffusion matrices for potassium and sodium channels. The $\mathbf{A}_{\mathrm{K}}$ and $\mathbf{A}_{\mathrm{Na}}$ drift matrices are

$$
\mathbf{A}_{\mathrm{K}}=\left[\begin{array}{cccc}
-\left(3 \alpha_{n}+\beta_{n}\right) & 2 \beta_{n} & 0 & 0 \\
3 \alpha_{n} & -2\left(\alpha_{n}+\beta_{n}\right) & 3 \beta_{n} & 0 \\
0 & 2 \alpha_{n} & -\left(\alpha_{n}+3 \beta_{n}\right) & 4 \beta_{n} \\
0 & 0 & \alpha_{n} & -4 \beta_{n}
\end{array}\right]
$$




$$
\mathbf{A}_{\mathrm{Na}}=\left[\begin{array}{ccccccc}
-\left(2 \alpha_{m}+\beta_{m}+\alpha_{h}\right) & 2 \beta_{m} & 0 & 0 & \beta_{h} & 0 & 0 \\
2 \alpha_{m} & -\left(\alpha_{m}+2 \beta_{m}+\alpha_{h}\right) & 3 \beta_{m} & 0 & 0 & \beta_{h} & 0 \\
0 & \alpha_{m} & -\left(3 \beta_{m}+\alpha_{h}\right) & 0 & 0 & 0 & \beta_{h} \\
0 & 0 & 0 & -\left(3 \alpha_{m}+\beta_{h}\right) & \beta_{m} & 0 & 0 \\
\alpha_{h} & 0 & 0 & 3 \alpha_{m} & -\left(2 \alpha_{m}+\beta_{m}+\beta_{h}\right) & 2 \beta_{m} & 0 \\
0 & \alpha_{h} & 0 & 0 & 2 \alpha_{m} & -\left(\alpha_{m}+2 \beta_{m}+\beta_{h}\right) & 3 \beta_{m} \\
0 & 0 & \alpha_{h} & 0 & 0 & \alpha_{m} & -\left(3 \beta_{m}+\beta_{h}\right)
\end{array}\right],
$$

and the $\mathbf{D}_{\mathrm{K}}^{\mathrm{eq}}$ and $\mathbf{D}_{\mathrm{Na}}^{\mathrm{eq}}$ diffusion matrices, in the equilibrium noise approximation, are

$$
\begin{aligned}
& \mathbf{D}_{\mathrm{K}}^{\mathrm{eq}}=\frac{1}{N_{\mathrm{K}}}\left[\begin{array}{cccc}
4 \alpha_{n} X_{0}^{0}+\left(3 \alpha_{n}+\beta_{n}\right) X_{1}^{0}+2 \beta_{n} X_{2}^{0} & -\left(3 \alpha_{n} X_{1}^{0}+2 \beta_{n} X_{2}^{0}\right) & 0 & 0 \\
-\left(3 \alpha_{n} X_{1}^{0}+2 \beta_{n} X_{2}^{0}\right) & 3 \alpha_{n} X_{1}^{0}+2\left(\alpha_{n}+\beta_{n}\right) X_{2}^{0}+3 \beta_{n} X_{3}^{0} & -\left(2 \alpha_{n} X_{2}^{0}+3 \beta_{n} X_{3}^{0}\right) & 0 \\
0 & -\left(2 \alpha_{n} X_{2}^{0}+3 \beta_{n} X_{3}^{0}\right) & 2 \alpha_{n} X_{2}^{0}+\left(\alpha_{n}+3 \beta_{n}\right) X_{3}^{0}+4 \beta_{n} X_{4}^{0} & -\left(\alpha_{n} X_{3}^{0}+4 \beta_{n} X_{4}^{0}\right) \\
0 & 0 & -\left(\alpha_{n} X_{3}^{0}+4 \beta_{n} X_{4}^{0}\right) & \alpha_{n} X_{3}^{0}+4 \beta_{n} X_{4}^{0}
\end{array}\right], \\
& \mathbf{D}_{\mathrm{Na}}^{\mathrm{eq}}=\frac{1}{N_{\mathrm{Na}}}\left[\begin{array}{ccccccc}
D_{11}^{\mathrm{eq}} & -2\left(\alpha_{m} Y_{10}^{0}+\beta_{m} Y_{20}^{0}\right) & 0 & 0 & -\left(\alpha_{h} Y_{10}^{0}+\beta_{h} Y_{11}^{0}\right) & 0 & 0 \\
-2\left(\alpha_{m} Y_{10}^{0}+\beta_{m} Y_{20}^{0}\right) & D_{22}^{\mathrm{eq}} & -\left(\alpha_{m} Y_{20}^{0}+3 \beta_{m} Y_{30}^{0}\right) & 0 & 0 & -\left(\alpha_{h} Y_{20}^{0}+\beta_{h} Y_{21}^{0}\right) & 0 \\
0 & -\left(\alpha_{m} Y_{20}^{0}+3 \beta_{m} Y_{30}^{0}\right) & D_{33}^{\mathrm{eq}} & 0 & 0 & 0 & -\left(\alpha_{h} Y_{30}^{0}+\beta_{h} Y_{31}^{0}\right) \\
0 & 0 & 0 & D_{44}^{\mathrm{eq}} & -\left(3 \alpha_{m} Y_{01}^{0}+\beta_{m} Y_{11}^{0}\right) & 0 & 0 \\
-\left(\alpha_{h} Y_{10}^{0}+\beta_{h} Y_{11}^{0}\right) & 0 & 0 & -\left(3 \alpha_{m} Y_{01}^{0}+\beta_{m} Y_{11}^{0}\right) & D_{55}^{\mathrm{eq}} & -2\left(\alpha_{m} Y_{11}^{0}+\beta_{m} Y_{21}^{0}\right) & 0 \\
0 & -\left(\alpha_{h} Y_{20}^{0}+\beta_{h} Y_{21}^{0}\right) & 0 & 0 & -2\left(\alpha_{m} Y_{11}^{0}+\beta_{m} Y_{21}^{0}\right) & D_{66}^{\mathrm{eq}} & -\left(\alpha_{m} Y_{21}^{0}+3 \beta_{m} Y_{31}^{0}\right) \\
0 & 0 & -\left(\alpha_{h} Y_{30}^{0}+\beta_{h} Y_{31}^{0}\right) & 0 & 0 & -\left(\alpha_{m} Y_{21}^{0}+3 \beta_{m} Y_{31}^{0}\right) & D_{77}^{\mathrm{eq}}
\end{array}\right],
\end{aligned}
$$

where the diagonal elements are

$$
\begin{aligned}
& D_{11}^{\mathrm{eq}}=3 \alpha_{m} Y_{00}^{0}+\left(2 \alpha_{m}+\beta_{m}+\alpha_{h}\right) Y_{10}^{0}+2 \beta_{m} Y_{20}^{0}+\beta_{h} Y_{11}^{0}, \\
& D_{22}^{\mathrm{eq}}=2 \alpha_{m} Y_{10}^{0}+\left(\alpha_{m}+2 \beta_{m}+\alpha_{h}\right) Y_{20}^{0}+3 \beta_{m} Y_{30}^{0}+\beta_{h} Y_{21}^{0}, \\
& D_{33}^{\mathrm{eq}}=\alpha_{m} Y_{20}^{0}+\left(3 \beta_{m}+\alpha_{h}\right) Y_{30}^{0}+\beta_{h} Y_{31}^{0}, \\
& D_{44}^{\mathrm{eq}}=\alpha_{h} Y_{00}^{0}+\left(3 \alpha_{m}+\beta_{h}\right) Y_{01}^{0}+\beta_{m} Y_{11}^{0}, \\
& D_{55}^{\mathrm{eq}}=\alpha_{h} Y_{10}^{0}+3 \alpha_{m} Y_{01}^{0}+\left(2 \alpha_{m}+\beta_{m}+\beta_{h}\right) Y_{11}^{0}+2 \beta_{m} Y_{21}^{0}, \\
& D_{66}^{\mathrm{eq}}=\alpha_{h} Y_{20}^{0}+2 \alpha_{m} Y_{11}^{0}+\left(\alpha_{m}+2 \beta_{m}+\beta_{h}\right) Y_{21}^{0}+3 \beta_{m} Y_{31}^{0}, \\
& D_{77}^{\mathrm{eq}}=\alpha_{h} Y_{30}^{0}+\alpha_{m} Y_{21}^{0}+\left(3 \beta_{m}+\beta_{h}\right) Y_{31}^{0} .
\end{aligned}
$$

Notation is the same as that used previously except that transmembrane potential dependence of the transition rates is not explicitly indicated.

\section{APPENDIX C: LINEAR SDE MODEL}

Obtaining linear SDE model predictions for transmembrane potential fluctuation statistics hinges on two computations: proper formulation of the Eq. (6) Jacobian matrix (note that $\mathbf{A}=-\mathbf{J}$ ) from the channel-based SDE model given by Eq. (3) and solving Eq. (7) for the covariance matrix elements, $\Sigma_{i j}$. Although both $\mathbf{J}$ and $\boldsymbol{\Sigma}$ are large matrices with $12 \times 12=144$ matrix elements, in each case some degree of simplification is possible. For the Jacobian matrix, J, the matrix elements are readily computed from the Eq. (6) definition from which it is found that 86 are identically zero and 58 are nonzero. Exact expressions for the Jacobian matrix elements and additional information related to formulation of the linear SDE can be found in the online Supplemental Material [35].

As noted in Sec. III, computation of the covariance matrix elements, $\Sigma_{i j}$, of the linearized channel-based SDE model via Eq. (7) was accomplished using a combination of symbolic and variable precision arithmetic in MATLAB 2014a with the Symbolic Math Toolbox (version 6.0). Starting with Eq. (7) and substituting for the drift and diffusion matrices we obtain

$$
\mathbf{J} \boldsymbol{\Sigma}+\boldsymbol{\Sigma} \mathbf{J}^{\mathrm{T}}+\left(\mathbf{S}^{\mathrm{eq}}\right)^{2}=0
$$

where we have taken advantage of the symmetry of the potassium and sodium diffusion matrices $\left[\mathbf{D}_{\mathrm{K}(\mathrm{Na})}\right], \mathbf{S}=$ $\operatorname{diag}\left(0, \mathbf{S}_{\mathrm{K}}, \mathbf{S}_{\mathrm{Na}}\right)$ and have explicitly indicated use of the equilibrium noise approximation. While at most this equation represents a system of $12 \times 12=144$ linear equations, symmetry of the covariance matrix, $\Sigma=\Sigma^{\mathrm{T}}$, reduces this system to 78 unknown covariance matrix elements. The algorithm used to solve this system is given in Table III. Note that because the Jacobian matrix is a function of the

\begin{tabular}{|c|c|}
\hline Step & Procedure \\
\hline 1 & For a given $I_{\mathrm{DC}}$, compute the steady-state vector $\mathbf{Z}^{0}$ \\
\hline 2 & $\begin{array}{l}\text { Numerically compute the } \mathbf{J} \text { and } \mathbf{S}^{\text {eq }} \text { matrices at the nominated } \\
\text { steady state }\end{array}$ \\
\hline 3 & $\begin{array}{l}\text { Using the sym (...) function, convert the } \mathbf{J} \text { and } \mathbf{S}^{\mathrm{eq}} \text { matrices to } \\
\text { symbolic form }\end{array}$ \\
\hline 4 & $\begin{array}{l}\text { Using the } \operatorname{vpa}(\ldots) \text { function, form the symbolic expression } \\
\qquad \mathbf{J} \boldsymbol{\Sigma}+\boldsymbol{\Sigma} \mathbf{J}^{\mathrm{T}}+\left(\mathbf{S}^{\mathrm{eq}}\right)^{2}\end{array}$ \\
\hline 5 & $\begin{array}{l}\text { Use vpasolve }(\ldots) \text { to compute each unique } \Sigma_{i j} \text { matrix } \\
\text { element }\end{array}$ \\
\hline 6 & Convert the symbolic $\Sigma_{i j}$ matrix elements to double precision \\
\hline
\end{tabular}
input current-dependent $\mathbf{Z}^{0}=\left[V^{0}, X_{1}^{0}, \ldots, X_{4}^{0}, Y_{10}^{0}, \ldots, Y_{31}^{0}\right]^{\mathrm{T}}$ steady state, the Jacobian matrix must be re-evaluated at each input current prior to formulating and solving the system of linear equations for the unknown covariance matrix elements. It is also important to note that implementation of the Table III algorithm in MATLAB 2013b results in spurious output due to a bug that we have traced to the vpasolve function. The use of MATLAB 2014a resolves this issue.

TABLE III. MATLAB algorithm for solving the Eq. (7) system of linear equations for the 78 unknown covariance matrix elements. 
[1] A. L. Hodgkin and A. F. Huxley, A quantitative description of membrane current and its application to conduction and excitation in nerve, J. Physiol. 117, 500 (1952).

[2] Alain Destexhe and Michelle Rudolph-Lilith, Neuronal Noise, Springer Series in Computational Neuroscience, Vol. 8 (Springer, New York, 2012).

[3] A. Aldo Faisal, Luc P. J. Selen, and Danial M. Wolpert, Noise in the nervous system, Nat. Rev. Neurosci. 9, 292 (2008).

[4] Elad Schneidman, Barry Freedman, and Idan Segev, Ion channel stochasticity may be critical in determining the reliability and precision of spike timing, Neural Comput. 10, 1679 (1998).

[5] Adam F. Strassberg and Louis J. DeFelice, Limitations of the Hodgkin-Huxley formalism: Effects of single channel kinetics on transmembrane voltage dynamics, Neural Comput. 5, 843 (1993).

[6] Eugene M. Izhikevich, Which model to use for cortical spiking neurons? IEEE T. Neural Networks 15, 1063 (2004).

[7] A. L. Hodgkin, The local electric changes associated with repetitive action in a non-medullated axon, J. Physiol. 107, 165 (1948).

[8] Eugene M. Izhikevich, Neural excitability, spiking and bursting, Int. J. Bifurcat. Chaos 10, 1171 (2000).

[9] Steven H. Strogatz, Nonlinear Dynamics and Chaos: With Applications to Physics, Biology, Chemistry, and Engineering (Westview Press, Cambridge, MA, 2000).

[10] Hermann Haken, Synergetics: Introduction and Advanced Topics (Springer, New York, 2004).

[11] W. Horsthemke and R. Lefever, Noise-Induced Transitions: Theory and Applications in Physics, Chemistry, and Biology (Springer, New York, 1983).

[12] D. A. Steyn-Ross, Moira L. Steyn-Ross, M. T. Wilson, and J. W. Sleigh, White-noise susceptibility and critical slowing in neurons near spiking threshold, Phys. Rev. E 74, 051920 (2006).

[13] G. Matsumoto and T. Kunisawa, Critical slowing-down near the transition region from the resting to time-ordered states in squid giant axons, J. Phys. Soc. Jpn. 44, 1047 (1978).

[14] Ronald F. Fox and Yan-nan Lu, Emergent collective behavior in large numbers of globally coupled independently stochastic ion channels, Phys. Rev. E 49, 3421 (1994).

[15] Thomas Nowotny and Mikhail I. Rabinovich, Dynamical origin of independent spiking and bursting activity in neural microcircuits, Phys. Rev. Lett. 98, 128106 (2007).

[16] Thomas Nowotny and Mikhail I. Rabinovich, Erratum: Dynamical origin of independent spiking and bursting activity in neural microcircuits, Phys. Rev. Lett. 101, 079901(E) (2008).

[17] R. D. Traub and R. Miles, Neural Networks of the Hippocampus (Cambridge University Press, New York, 1991).

[18] Michael R. Guevara, Nonlinear Dynamics in Physiology and Medicine, Dynamics of Excitable Cells edited by Anne Beuter, Leon Glass, Michael C. Mackey, and Michele S. Titcombe, Interdisciplinary Applied Mathematics, Vol. 25 (Springer, New York, 2003).
[19] R. Fitzhugh, A kinetic model of the conductance changes in nerve membrane, J. Cell. Compar. Physiol. Suppl. 66, 111 (1965).

[20] Joshua H. Goldwyn and Eric Shea-Brown, The what and where of adding channel noise to the Hodgkin-Huxley equations, PLOS Comput. Biol. 7, e1002247 (2011).

[21] Joshua H. Goldwyn, Nikita S. Imennov, Michael Famulare, and Eric Shea-Brown, Stochastic differential equation models for ion channel noise in Hodgkin-Huxley neurons, Phys. Rev. E 83, 041908 (2011).

[22] Peter Rowat, Interspike interval statistics in the stochastic Hodgkin-Huxley model: Coexistence of gamma frequency bursts and highly irregular firing, Neural Comput. 19, 1215 (2007).

[23] Daniele Linaro, Marco Storace, and Michele Giugliano, Accurate and fast simulation of channel noise in conductance-based model neurons by diffusion approximation, PLoS Comput. Biol. 7, e1001102 (2011).

[24] Crispin Gardiner, Stochastic Methods: A Handbook for the Natural and Social Sciences, 4th ed. (Springer, Berlin, 2009).

[25] Daniel T. Gillespie, Exact stochastic simulation of coupled chemical reactions, J. Phys. Chem. 81, 2340 (1977).

[26] Carson C. Chow and John A. White, Spontaneous action potentials due to channel fluctuations, Biophys. J. 71, 3013 (1996).

[27] E. Skaugen and L. Walløe, Firing behaviour in a stochastic nerve membrane model based upon the Hodgkin-Huxley equations, Acta Physiol. Scand. 107, 343 (1979).

[28] Peter E. Kloeden and Eckhard Platen, Numerical Solution of Stochastic Differential Equations, Applications of Mathematics, Vol. 23 (Springer, New York, 1999).

[29] Walter J. Freeman, NDN, volume transmission, and selforganization in brain dynamics, J. Integr. Neurosci. 4, 407 (2005).

[30] Hugh R. Wilson, Simplified dynamics of human and mammalian neocortical neurons, J. Theor. Biol. 200, 375 (1999).

[31] Carlo Laing and Gabriel J Lord, eds., Stochastic Methods in Neuroscience (Oxford University Press, New York, 2010).

[32] M. A. D. Roa, M. Copelli, O. Kinouchi, and N. Caticha, Scaling law for the transient behavior of type-II neuron models, Phys. Rev. E 75, 021911 (2007).

[33] C. Meisel, A. Klaus, C. Kuehn, and D. Plenz, Critical slowing down governs the transition to neuron spiking, PLOS Comput. Biol. 11(2), 1004097 (2015).

[34] David F. Anderson, Bard Ermentrout, and Peter J. Thomas, Stochastic representations of ion channel kinetics and exact stochastic simulation of neuronal dynamics, J. Comput. Neurosci. 38, 67 (2015).

[35] See Supplemental Material at http://link.aps.org/supplemental/ 10.1103/PhysRevE.91.032708 for a detailed description of the linear SDE model. 